\title{
Fuerza lumbar en jugadores de hockey hierba
}

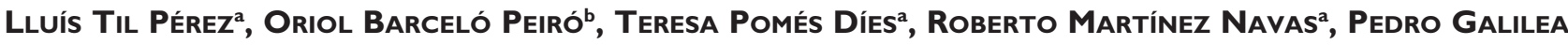 Ballarini $^{c}$ y Montserrat Bellver Vives ${ }^{a}$}

aUnidad Asistencial del Consorcio Sanitario de Terrassa en el Centro de Alto Rendimiento de Sant Cugat. Sant Cugat del Vallès. Barcelona. España. 'Medicina del Deporte del Centro de Alto Rendimiento de Sant Cugat. Escuela de Medicina de la Educación Física y del Deporte de Barcelona. Universidad de Barcelona. Barcelona. España.

'Departamento de Fisiología del Centro de Alto Rendimiento de Sant Cugat del Vallès. Sant Cugat del Vallès. Barcelona. España.

\section{RESUMEN}

Introducción: El dolor lumbar tiene una alta prevalencia entre los deportistas, se ha relacionado con déficits en la fuerza extensora lumbar, y el hecho de padecerlo representa un obstáculo importante para la práctica de deportes de alta intensidad.

Método: Se ha medido la fuerza lumbar en 2 grupos de practicantes de hockey hierba mediante máquina MedX ${ }^{\circledR}$ y un test de resistencia isométrico lumbar.

Resultados: Entre ambos grupos los resultados han sido muy homogéneos. Los 2 tests no presentan relación entre sí ni con las características biomédicas de los jugadores (edad, índice de masa corporal $\circ \mathrm{VO}_{2 \text { máx }}$ ). Los niveles de fuerza máxima y resistencia isométrica obtenidos han sido superiores a los de referencia entre sedentarios. Característicamente, entre los jugadores de hockey hierba la relación entre la fuerza extensora del tronco en flexión (M) respecto a la fuerza extensora del tronco en extensión $(\mathrm{m})$ es mayor que en otros deportistas (ratio $\mathrm{M} / \mathrm{m}>1,6$, mientras que en la población normal es I,4) debido probablemente a la postura en semiflexión propia del hockey.

Conclusión: Los resultados de los test de fuerza extensora lumbar tienen unas características propias entre los jugadores de hockey hierba.

PALABRAS CLAVE: Hockey hierba. Lumbalgia. Test de fuerza. Fuerza máxima. Fuerza resistencia.

\section{ABSTRACT}

Introduction: The prevalence of lower back pain is high among sportsmen. Lower back pain is related to deficiencies in lumbar extensor strength and lack of this strength is a substantial handicap for practicing high intensity sports.

Methods: Lumbar strength was evaluated in 2 groups of field hockey through the Med ${ }^{\circledR}$ machine and a lumbar isometric endurance test.

Results: The results were highly homogenous between the two groups. No associations were found between the 2 tests or with player characteristics (age, body mass index, $\left.\mathrm{VO}_{2 \max }\right)$. Peak torque and isometric endurance were higher than reference values among the sedentary population. Field hockey players characteristically had greater trunk flexion strength and lower extension strength than the general population $(\mathrm{M} / \mathrm{m}$ ratio $>1.6$, compared with a normal value of I.4) probably due to the characteristic hockey position of semiflexion.

Conclusion: The lumbar strength test is a useful screening method to identify individuals at risk of lower back pain.

KEY WORDS: Field hockey. Low back pain. Strength test. Peak torque. Endurance. 


\section{INTRODUCCIÓN}

La lumbalgia representa un elevado coste sanitario y social. En su origen intervienen factores orgánicos, laborales y psicosociales. La prevalencia de la lumbalgia durante tota la vida es del 60 al 80\%, con una tasa de incidencia anual del 5 al 25\%

Entre los deportistas el dolor lumbar comporta entre el 10 y el $20 \%$ de sus lesiones. Tiene importantes implicaciones en su rendimiento, y condiciona su participación en entrenamientos y en la competición. También puede afectar de forma notable a la obtención de sus objetivos deportivos ${ }^{2,3}$.

Uno de los factores de riesgo más relacionados con la lumbalgia es la disfunción de la musculatura del tronco ${ }^{4}$ y el descenso de la capacidad de resistencia de la musculatura extensora lumbar ${ }^{5,6}$. Una herramienta importante para la prevención y la rehabilitación de la lumbalgia es el trabajo activo de la musculatura extensora lumbar.

Entre los diferentes sistemas para valorar la fuerza muscular del tronco se encuentran los modelos isocinéticos, que son complejos y están poco implantados, y los modelos isométricos instrumentalizados, como el que se realiza en el dinamómetro de extensión lumbar MedX $\mathrm{X}^{8}$. Hay también otros métodos que pretenden valorar la resistencia isométrica de la musculatura del tronco, como el test de Sorensen-Biering (TSB) (para los músculos extensores del tronco) o la prueba de Shirado (para los músculos flexores del tronco) $)^{5,6,9}$.

Los jugadores de hockey hierba, como la mayoría de deportistas, forman parte de la población de riesgo para padecer lumbalgia. Desarrollan una buena parte del juego en semiflexión asimétrica y los cambios de ritmo son frecuentes. Está bien documentada la lesionabilidad de sus músculos isquiosurales, detalle que algunos autores han relacionado con disfunción lumbar ${ }^{3}$.

\section{Objetivos}

Medir la fuerza extensora lumbar de los jugadores de hockey hierba de 2 equipos diferentes y en diferentes momentos de competición, por medio de 2 tests de fuerza lumbar:

- Test de Sorensen-Biering (TSB), test isométrico de la musculatura extensora lumbar (fig. 1).

- Test de fuerza máxima isométrica en 7 puntos de rango de movimiento (ROM) mediante máquina $\mathrm{MedX}{ }^{\circledR}$ (fig. 2).

Con los datos obtenidos se quiere valorar:

- Si los jugadores de los 2 equipos presentan el mismo patrón de fuerza isométrica extensora lumbar en máquina

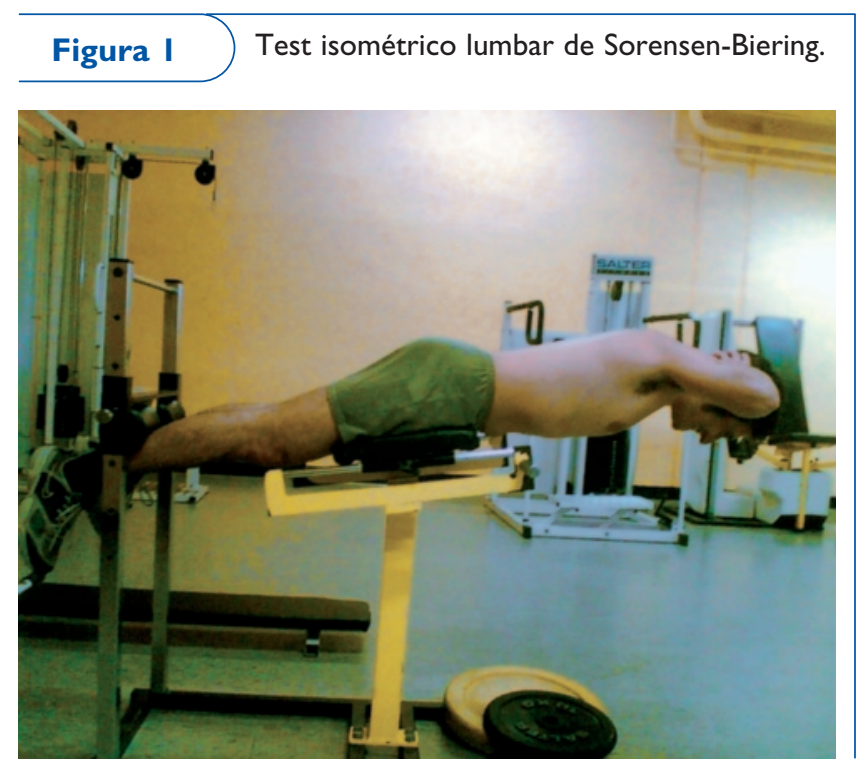

\section{Figura 2 Test de fuerza máxima isométrica en $\operatorname{Med}^{\circledR}$.}

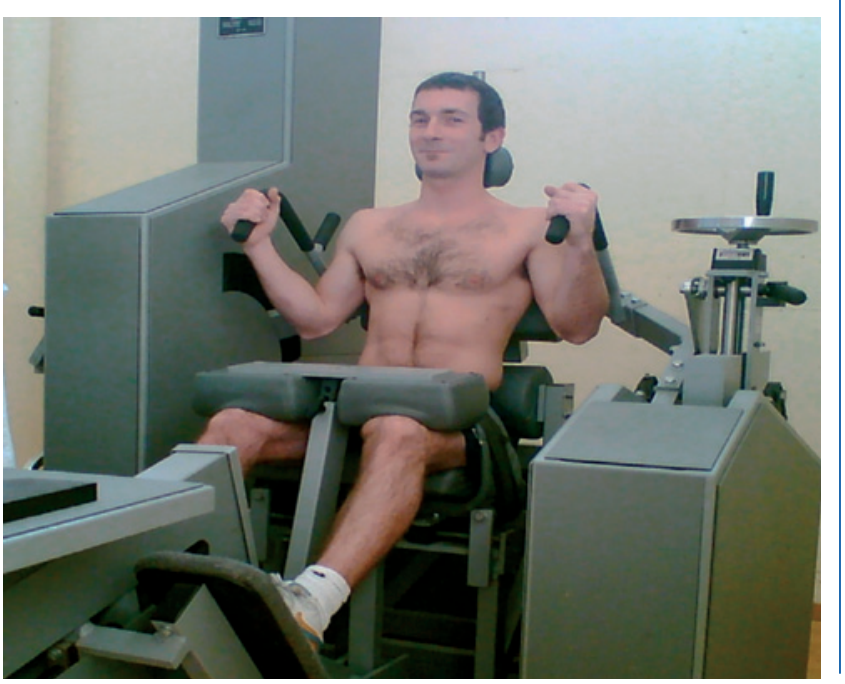

$\mathrm{MedX}^{\circledR}$. Si existiera este patrón propio de los jugadores de hockey hierba, cuál es la diferencia respecto al patrón normal y al patrón de deportistas del Centro de Alto Rendimiento.

- Si hay relación estadística entre los 2 tests (entre el TSB y las diferentes variables obtenidas en la máquina $\left.\mathrm{MedX}{ }^{\circledR}\right)$ : fuerza máxima, sumatoria de las fuerzas máximas y ratio $\mathrm{M} / \mathrm{m}$ (cociente entre la fuerza máxima y mínima).

- Si hay relación estadística entre el TSB y las variables propias de la población (edad, índice de masa corporal [IMC] y $\left.\mathrm{VO}_{2 \max }\right)$. 


\section{MÉTOdOS}

\section{Población}

Se valoran 26 jugadores de hockey hierba masculinos de 2 equipos diferentes:

Grupo 1: 14 jugadores de la selección estatal en fase de preparación del campeonato del mundo, valorados durante los meses de febrero y marzo de 2003. Edad: $24 \pm 4,25$ años; peso: $77,4 \pm 4,93 \mathrm{~kg}$; altura: $1,81 \pm 0,05 \mathrm{~m} ; \mathrm{VO}_{2 \text { máx }}: 58 \pm 3,0$ $\mathrm{ml} / \mathrm{kg} / \mathrm{min}$.

Grupo 2: 12 jugadores de un equipo de primera división de la liga española, a los que se les va a realizar los test durante su temporada regular, en noviembre de 2006. Edad: $22 \pm 3,63$ años; peso: $73 \pm 7,4 \mathrm{~kg}$; altura: $1,77 \pm 0,05 \mathrm{~m}$; $\mathrm{VO}_{2 \max }: 57,5 \pm$ $4,4 \mathrm{ml} / \mathrm{kg} / \mathrm{min}$.

Se informa a los participantes de la naturaleza de las pruebas y de los procedimientos que se realizan, y se obtiene el consentimiento informado por escrito de todos ellos.

\section{Procedimiento}

A los jugadores del grupo 1 se les realiza un test isométrico lumbar en máquina $\mathrm{MedX}{ }^{\circledR}$, según protocolo convencional. El sistema $\mathrm{MedX}^{\circledR}$ está equipado con un dinamómetro capaz de medir la fuerza estática y dinámica extensora lumbar. Después de la sesión de calentamiento y familiarización, de $90 \mathrm{~s}$ de duración, isotónico y que consiste en 10 repeticiones con $20 \mathrm{~kg}$, se realiza el test isométrico en 7 puntos de ángulo del ROM, empezando en $72^{\circ}$ (máxima flexión del tronco) y terminando en $0^{\circ}$ (máxima extensión del tronco). Mediante soportes específicos se estabilizan las extremidades y la pelvis en el asiento, y de esta forma se mide de forma aislada el trabajo de la musculatura específicamente extensora lumbar. Durante el test los sujetos son estimulados para conseguir la fuerza máxima. Los resultados obtenidos se recogen en un software que permite la evaluación posterior ${ }^{4,10,11}$. Se trata de un test poco confortable pero muy específico, y requiere personal entrenado.

A los jugadores del grupo 2 se efectúa la historia de su patología lumbar. A cada individuo se le practican 3 test clínicos específicos. En primer lugar el test de Adams: en bipedestación y sin flexionar las rodillas, se realiza una flexión del tronco con los brazos extendidos, para descartar rotaciones del tronco secundarias a escoliosis. En segundo lugar se mide la longitud de las extremidades inferiores, para descartar disimetrías. Y en último lugar se mide el ángulo poplíteo de cada rodilla: en decúbito supino con la cadera a $90^{\circ}$ de flexión en rotación neutra, y se mide el ángulo complementario a la máxima extensión pasiva de la rodilla. A continuación, antes de la realización del test TSB, los individuos realizan ejercicios de calentamiento, sin carga, de la musculatura lumbar (5 repeticiones) para reducir el riesgo de lesiones. El TSB explora la resistencia isométrica de los músculos extensores del tronco (musculatura lumbar). El paciente se coloca en decúbito prono sobre un banco de trabajo lumbar, con la pelvis y los tobillos fijados y el tronco libre a partir de las espinas ilíacas anterosuperiores. Con las manos en la nuca, el sujeto ha de mantener la horizontalidad del cuerpo efectuando una contracción isométrica de la musculatura lumbar el mayor tiempo posible (cronometrado). Los individuos se instruyen para mantener la posición tanto como les sea posible, y durante el test reciben un estímulo si la posición se sitúa por debajo del nivel horizontal. La posición horizontal se controla por una plomada colgante que se coloca rozando su espalda, lo que permite al individuo darse cuenta de si mantiene la horizontalidad (fig. 1).

Si la posición no se corrige rápidamente, o si el individuo claudica la posición por fatiga o por incomodidad, el test se considera finalizado y se toma nota del tiempo ${ }^{5,6}$.

Una vez finalizado el test, después de un período de recuperación de 15 min los individuos inician un test isométrico lumbar en 7 puntos a la MedX ${ }^{\circledR}$, de la misma manera que el que realizaron los individuos del grupo 1 .

Los valores obtenidos en $\mathrm{MedX}^{\circledR}$ son:

- El promedio de la fuerza máxima obtenida (absoluta y relativa al peso corporal).

- Suma de las fuerzas obtenidas en los 7 puntos del ROM.

- La ratio $\mathrm{M} / \mathrm{m}$ (cociente entre la fuerza máxima y mínima en los 7 puntos del ROM).

- Promedios de la fuerza obtenida en cada uno de los 7 puntos del ROM.

Se reproducen las medias y las desviaciones estándar de todas las variables estudiadas.

Para la comparación de las variables entre los 2 grupos de estudio se ha utilizado una prueba de homogeneidad de variancias, y la prueba de la t de Student. Para estudiar la correlación entre las variables del estudio se utiliza el coeficiente de correlación lineal de Pearson. El grado de significación se establece en $\mathrm{p}<0,05$.

\section{Resultados}

\section{Grupo I (tabla I y fig. 3)}

Con los resultados recogidos de la máquina $\mathrm{MedX}^{\circledR}$ se obtienen diferentes parámetros: 
Tabla Media y desviación estándar de la fuerza relativa y absoluta del grupo I en la MedX ${ }^{\circledR}$

\begin{tabular}{|lccccccc|}
\hline & $0^{\circ}$ & $12^{\circ}$ & $\mathbf{2 4}^{\circ}$ & $\mathbf{3 6}^{\circ}$ & $\mathbf{4 8}^{\circ}$ & $\mathbf{6 0}^{\circ}$ & $\mathbf{7 2}^{\circ}$ \\
\hline Relativa & $2,75 \pm 0,47$ & $3,34 \pm 0,46$ & $3,76 \pm 0,6$ & $3,85 \pm 0,79$ & $4,04 \pm 0,81$ & $4,45 \pm 0,94$ & $4,59 \pm 0,95$ \\
\hline Absoluta & $213 \pm 40$ & $259 \pm 46$ & $291 \pm 49,5$ & $298 \pm 65,5$ & $313 \pm 69$ & $346 \pm 83,5$ & $356 \pm 85$ \\
\hline
\end{tabular}

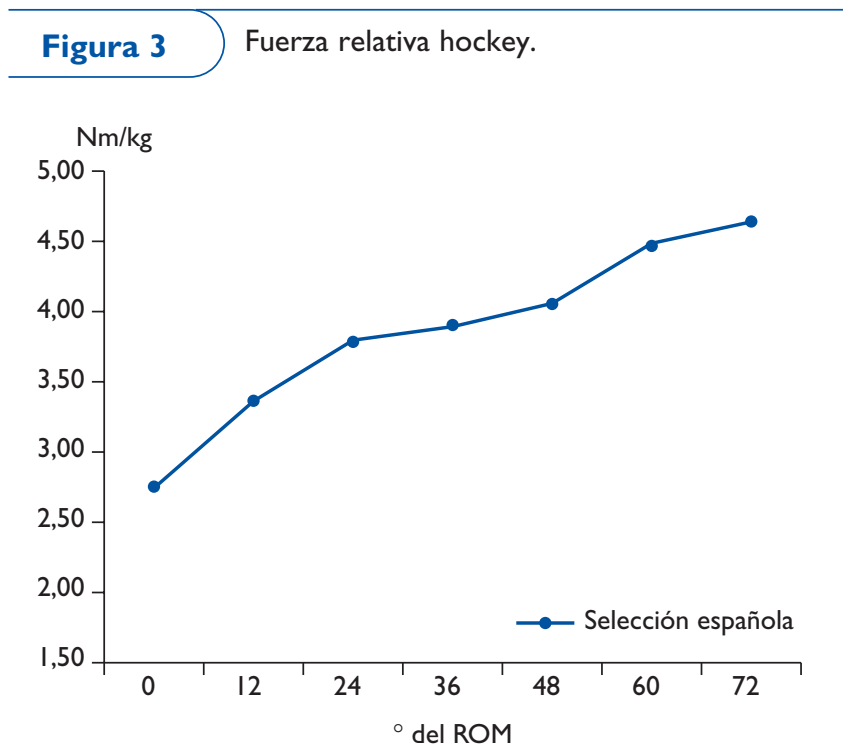

- La fuerza máxima absoluta es $358 \pm 84,3 \mathrm{Nm}$.

- La fuerza máxima relativa al peso corporal es de 4,61 \pm $0,94 \mathrm{Nm} / \mathrm{kg}$ de peso.

- La suma de las fuerzas máximas absolutas obtenidas en los 7 puntos del ROM es de 21.272 $\pm 4.085 \mathrm{Nm}$.

- La suma de las fuerzas máximas relativas obtenidas en los 7 puntos del ROM es de $26,77 \pm 4,59 \mathrm{Nm} / \mathrm{kg}$ de peso.

- La ratio $\mathrm{M} / \mathrm{m}$ (cociente entre la fuerza máxima y mínima en los 7 puntos del ROM) es de 1,68.

\section{Grupo 2 (tabla II y fig. 4)}

En la recogida de datos sobre la historia de lumbalgias, 5 jugadores refieren haber presentado episodios de lumbalgia en el último año, sin detectar ninguna alteración del patrón de fuerza respecto al resto del grupo.

En la exploración y valoración dorso-lumbar-pélvica se obtienen los siguientes resultados:

- Test d'Adams: en el 67\% se observa simetría dorsal, en el $17 \%$ hay elevación dorsal derecha, y también en el 17\% hay elevación dorsal izquierda, todas inferiores a $6^{\circ}$.

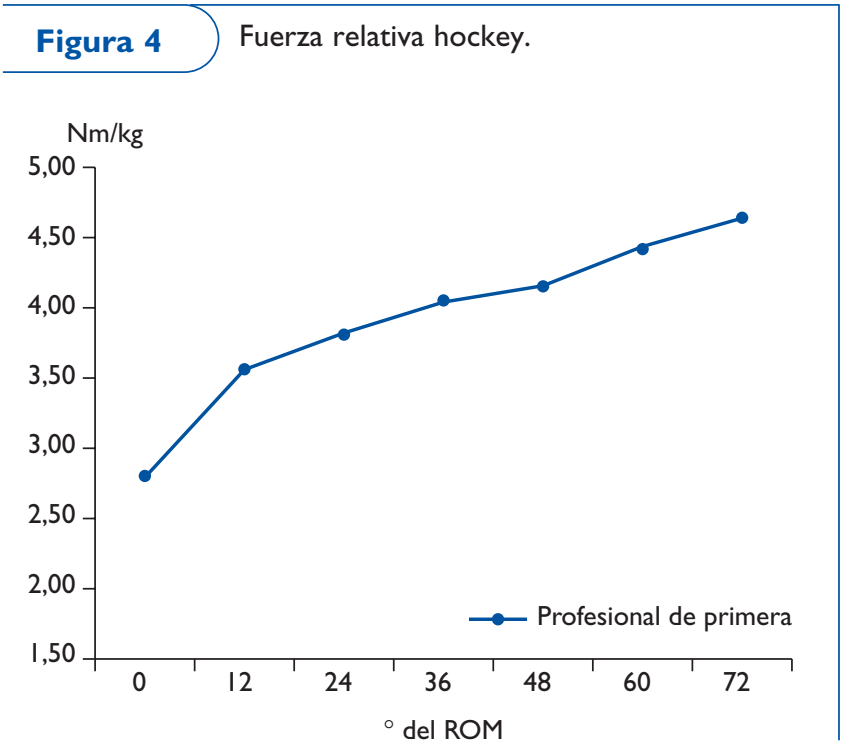

- Báscula pélvica: En el 84\% se observa una báscula pélvica equilibrada, en el $8 \%$, elevación de la espina ilíaca posterosuperior derecha y también en un $8 \%$ elevación de la espina ilíaca posterosuperior izquierda, en ningún caso superior a $8^{\circ}$.

- Ángulo poplíteo: El ángulo derecho es de $136,8 \pm 7,6^{\circ}$, y el ángulo izquierdo es de $135,3 \pm 10,2^{\circ}$.

En ninguno de los jugadores con test de Adams positivo o disimetría pélvica se observa alteración del patrón de fuerza respecto al resto del grupo, y tampoco respecto del ángulo poplíteo.

En el TSB, la media y la desviación estándar de los valores cronometrados del grupo es de $138 \pm 18,9$ s.

En la MedX ${ }^{\circledR}$ se obtienen diferentes valores:

- La fuerza máxima absoluta es de $340 \pm 73,5 \mathrm{Nm}$.

- La fuerza máxima relativa al peso corporal es de 4,65 \pm $0,79 \mathrm{Nm} / \mathrm{kg}$ de peso.

- La suma de las fuerzas máximas absolutas obtenidas en los 7 puntos del ROM es de $20.413 \pm 5.320 \mathrm{Nm}$.

- La suma de las fuerzas máximas relativas obtenidas en los 7 puntos del ROM es de 27,12 $\pm 5,82 \mathrm{Nm} / \mathrm{kg}$ de peso.

- La ratio $\mathrm{M} / \mathrm{m}$ (cociente entre la fuerza máxima y mínima) en los 7 puntos es de 1,68. 
TRABAJOS ORIGINALES

Tabla II Media y desviación estándar de la fuerza relativa al peso del grupo 2 en la MedX ${ }^{\circledR}$

\begin{tabular}{|lccccccc|}
\hline & $\mathbf{0}^{\circ}$ & $\mathbf{1 2}^{\circ}$ & $\mathbf{2 4}^{\circ}$ & $\mathbf{3 6}^{\circ}$ & $\mathbf{4 8}^{\circ}$ & $\mathbf{6 0}^{\circ}$ & $\mathbf{7 2}^{\circ}$ \\
\hline Relativa & $2,77 \pm 0,79$ & $3,51 \pm \mathrm{I}$ & $3,76 \pm \mathrm{I}$ & $3,99 \pm 0,95$ & $4,10 \pm 0,76$ & $4,38 \pm 0,7$ & $4,6 \mathrm{I} \pm 0,74$ \\
\hline Absoluta & $205 \pm 75$ & $259 \pm 91,5$ & $277 \pm 95,3$ & $293 \pm 84,3$ & $300 \pm 69,1$ & $320 \pm 72,9$ & $337 \pm 68,7$ \\
\hline
\end{tabular}

\section{CONCLUSIONES}

En los 2 grupos de hockey hierba se estudia si existen diferencias significativas entre las medias de las variables, con los siguientes resultados: edad, 1,92 (NS); peso, 4,36 (NS); IMC, 0,16 (NS); $\mathrm{VO}_{2 \text { máx }}, 0,5$ (NS); talla, 0,05 ( $\left.\mathrm{p}<0,05\right)$. Por tanto, puede concluirse que los 2 grupos son casi totalmente homogéneos (excepto la talla).

Se trata de un test practicado en 2 momentos diferentes de competición (febrero-marzo de 2003 y noviembre de 2006), pero dado que la condición física en el hockey hierba se mantiene durante tota la temporada y por tanto no influye en los resultados, podemos asumir que el resultado promedio es un patrón de fuerza para los jugadores de élite de hockey hierba en máquina $\operatorname{MedX}{ }^{\circledR}$.

Los 2 equipos presentan una media de la fuerza máxima similar. Los valores de fuerza máxima absoluta y relativa al peso de ambos grupos son un poco superiores a los valores obtenidos en el punto teórico de fuerza máxima ( $\mathrm{ROM} 72^{\circ}$ ), pues algunos jugadores obtienen el valor máximo en un punto inferior $\left(\mathrm{ROM} 60^{\circ}\right)$.

La ratio $\mathrm{M} / \mathrm{m}$ mantiene el mismo valor en los 2 grupos ( $r a-$ tio $\mathrm{M} / \mathrm{m}: 1,68)$, y este índice orienta sobre la pendiente de la curva. La ratio $\mathrm{M} / \mathrm{m}$ para sujetos no deportistas y para los deportistas del Centro de Alto Rendimiento (CAR) ( $\mathrm{n}>500$ testados) durante los últimos 7 años (ratio $\mathrm{M} / \mathrm{m}: 1,4$ ) proporciona la idea de que la fuerza de los extensores es un $40 \%$ superior en máxima flexión que en máxima extensión (tabla III). Esto se atribuye al pretensado de las estructures contráctiles y no contráctiles en flexión, al aumento de la presión abdominal en flexión y a la menor eficacia contráctil de los sarcómeros ya acortados en extensión. La ratio aumentada en los jugadores de hockey hierba $(1,68)$ (fig. 5) se debe a que ellos trabajan mucho tiempo en flexión y semiflexión, y por ello el grupo muscular es más eficaz aún en esta posición (fig. 6).

La suma de las fuerzas en los diferentes puntos del ROM, que algunos autores han asimilado a un área bajo la curva y, por tanto, a la capacidad de desarrollar trabajo, son casi iguales en ambos grupos, a pesar de que se encuentran por debajo de la media de los deportistas testados en el CAR, aunque ambos grupos se hallan muy por encima del patrón de fuerza considerado como normal entre sujetos sedentarios voluntarios.

No hay ninguna correlación significativa entre el test de Sorensen-Biering (TSB) y las diferentes variables obtenidas del test en máquina $\mathrm{MedX}{ }^{\circledR}$. El tiempo que un individuo es capaz de mantener la posición solicitada en el TSB realizando una contracción isométrica y a la vez isotónica mide la resistencia a la fatiga en contracción estática. Por otro lado, el test isométrico en máquina MedX valora de manera directa fuerzas máximas en 7 puntos de ROM diferentes, y de forma indirecta proporciona un índice de capacidad de trabajo y la relación entre la fuerza en diferentes posiciones del ROM, y estos resultados no tienen necesariamente que estar relacionados, en sujetos sanos y asintomáticos, con la resistencia a la fatiga del mismo grupo muscular.

Tabla III Media de la fuerza relativa máxima de los jugadores de hockey hierba, de los deportistas del Centro de Alto Rendimiento (CAR) y sedentarios en la MedX ${ }^{\circledR}$

\begin{tabular}{|llllllllll|}
\hline & $\mathbf{0}^{\circ}$ & $\mathbf{1 2}^{\circ}$ & $\mathbf{2 4}^{\circ}$ & $\mathbf{3 6}^{\circ}$ & $\mathbf{4 8}^{\circ}$ & $\mathbf{6 0}^{\circ}$ & $\mathbf{7 2}^{\circ}$ & $\mathbf{R a t i o}^{\circ}$ \\
\hline Sedentarios & 3,00 & 3,10 & 3,32 & 3,56 & 3,78 & 4,06 & 4,2 & I,40 \\
\hline CAR & 3,70 & 4,07 & 4,31 & 4,56 & 4,64 & 4,90 & 5,20 & I,4I \\
\hline Hockey hierba & 2,76 & 3,42 & 3,76 & 3,92 & 4,07 & 4,42 & 4,60 & 1,68 \\
\hline
\end{tabular}




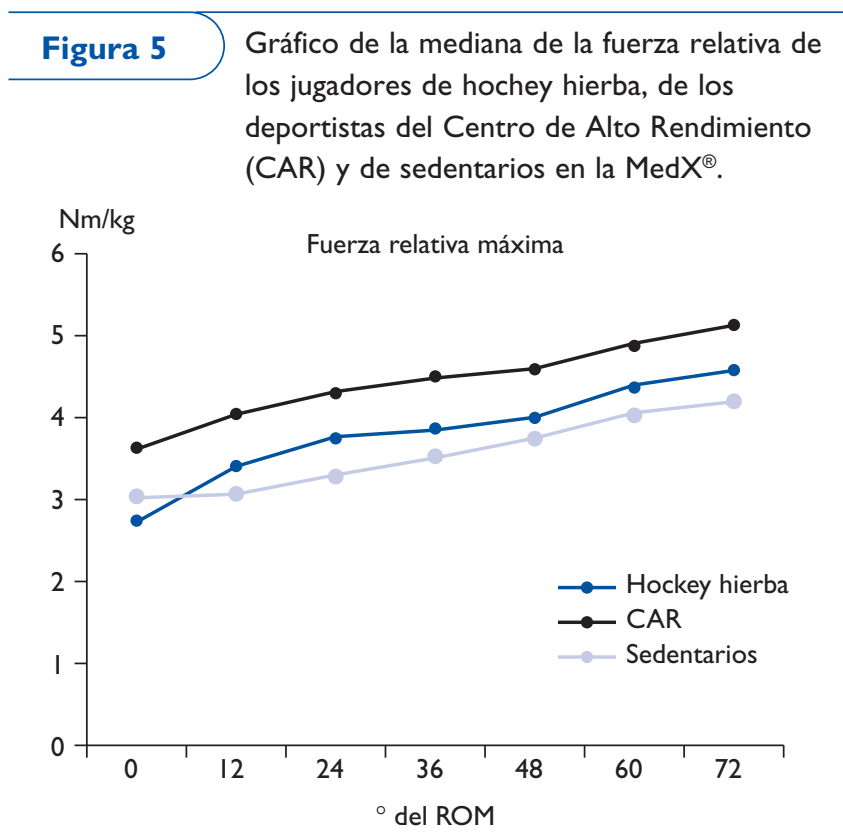

No hay correlación significativa alguna entre el TSB y las variables poblacionales edad, IMC o $\mathrm{VO}_{2 \max }$.

Los resultados del test de Sorensen-Biering (TSB) ponen de manifiesto que los individuos que mantienen la posición durante más de $60 \mathrm{~s}$ están protegidos ante la posibilidad de padecer lumbalgias durante el próximo año, y los autores que describen el test añaden que un individuo sano es capaz de mantener la posición al menos $120 \mathrm{~s}$. Todos los jugadores de hockey hierba sobrepasan este límite, y por ello consideramos valores normales para esta población $138 \pm 18,9 \mathrm{~s}$.

Hay que remarcar la idoneidad del TSB y de la máquina MedX ${ }^{\circledR}$ como métodos de cribado para detectar deportistas

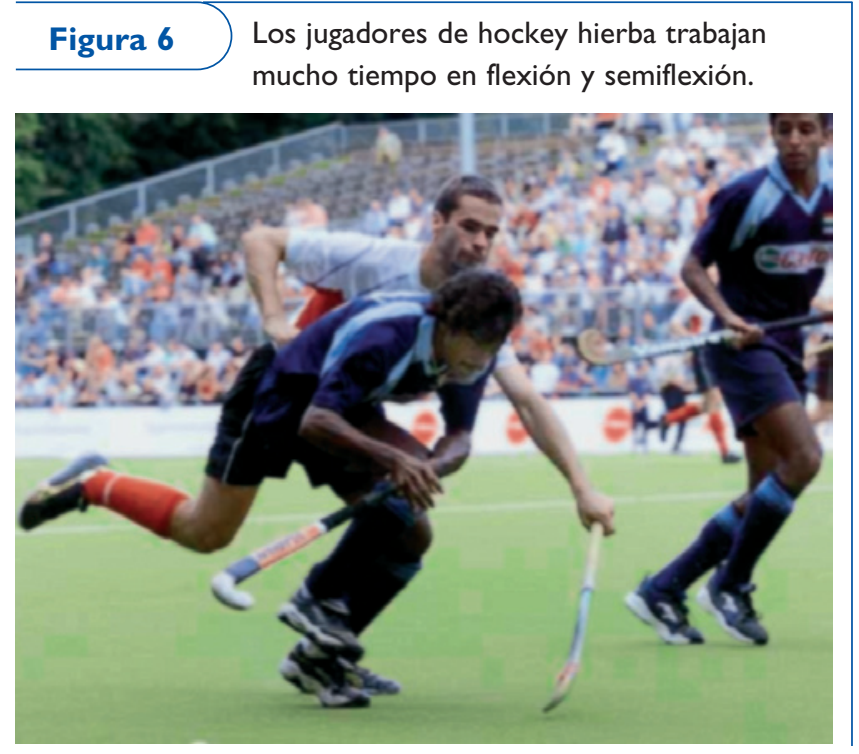

con disfunción de la musculatura lumbar, y que por tanto se encuentran en riesgo de padecer patología lumbar. Tanto la resistencia de la fuerza como la fuerza máxima son características entrenables, y por tanto puede afirmarse que este cribado permite detectar una susceptibilidad de padecer patología de la musculatura extensora lumbar y convertir la indicación de trabajo específico en prevención ${ }^{12}$.

\section{Agradecimientos}

A los servicios médicos y a los jugadores de hockey hierba de la selección española 2003 y del equipo Athlètic Terrassa 2006.

\section{Bibliografía}

1. Poiraudeau S, Lefevre-Colau M, Fayad F, Rannou F, Revel M. Lumbalgias. En: Enciclopedia Médico Quirúrgica. Aparato locomotor. París: Elsevier; 2004. E-14-549. 17.

2. Coumans JV, Lauerman WC. Head and Spine. En: Miller MD, Cooper DE, Warner JJP, editors. Review of sports medicine and arthroscopy. Philadelphia: Saunders; 2002. p. 262-9.

3. Nadler SF, Wu KD, Galski T, Feinberg JH. Low back pain in college athletes. A prospective study correlating lower extremity overuse or acquired ligamentous laxity with low back pain. Spine. 1998;23:828-33.

4. Carpenter DM, Nelson BW. Low back strengthening for the prevention and treatment of low back pain. Med Sci Sports Exerc. 1999;31:18-24.

5. Ropponen A, Gibbons LE, Videman T, Battié MC. Isometric back extension endurance testing: reasons for test termination. J Orthop Sports Phys Ther. 2005;35:437-42. 
6. Biering-Sorensen F. Physical measuraments as risk indicators for low-back troubleover a one-year period. Spine. 1984;9: 106-19.

7. García Pérez F, Alcántara-Bumbiedro S. Importancia del ejercicio físico en el tratamiento del dolor lumbar inespecífico. Rehabilitación (Madrid). 2003;7:323-32.

8. Mayer T, Gatchel R, Betancur J, Boyasso E. Trunk muscle endurance measurement. Isometric contrasted to isokinetic testing in normal subjects. Spine.1995;20:920-6.

9. Meier JL, Kerkour K, Mansuy J. Techniques de musculation abdominale et spinale. En: Encycl Méd Chir Kinésithérapie -
Rééducation fonctionnelle. Paris: Elsevier; 1996. 26-062-A$1014 \mathrm{p}$.

10. Hager SM, Udermann BE, Reineke DM, Gibson MH, Mayer JM, Murray SR. Quantification of lumbar endurance on a backup lumbar extension dynamometer. J Sports Sci Medicine. 2006;5:656-61.

11. Udermann BE, Mayer JM, Murray SR. Quantification of isometric lumbar extension strenght using a backup lumbar extension dynamometer. Res Q Exerc Sport. 2004;75:434-39.

12. Jorgensen K. Human trunk extensor muscles physiology and ergonomics. Acta Physiol Scand Suppl. 1997;637:1-58. 